\title{
Miniesternotomia e mini-incisão: experiência inicial do Instituto Dante Pazzanese de Cardiologia
}

\author{
Fabian CASTILHO*, Antoninho Sanfins ARNONI*, Renato T. ARNONI*, José Antônio RIVERA*, \\ Antônio Flávio Sanches de ALMEIDA*, Camilo ABDULMASSIH NETO*, Jarbas J. DINKHUYSEN*, \\ Mário ISSA*, Paulo CHACCUR* ${ }^{*}$ Paulo Paredes PAULISTA*
}

RBCCV 44205-485

Castilho F, Arnoni A S, Arnoni R T, Rivera J A, Almeida A F S, Abdulmassih Neto C, Dinkhuysen J J, Issa M, Chaccur P, Paulista P P - Miniesternotomia e mini-incisão: experiência inicial do Instituto Dante Pazzanese de Cardiologia. Rev Bras Cir Cardiovasc 2000; 15 (1): 39-43.

RESUMO: Objetivo: Como opção para abordagem cirúrgica do coração temos a miniesternotomia e a mini-incisão, sendo a última caracterizada por uma pequena abertura na pele com secção mediana total do esterno. O objetivo deste trabalho é avaliar estas duas opções de abordagem do coração quanto a viabilidade, reprodutividade e efeito estético final.

Casuística e Métodos: Foram realizadas miniesternotomias em "H" e em "L" e operações através de miniincisão para tratamento de valvopatias (aórtica e mitral), cardiopatias congênitas (CIV e CIA) e revascularização do miocárdio. Analisamos 35 pacientes, sendo 10 (40\%) submetidos a miniesternotomia e $25(60 \%)$ a mini-incisão. A idade média foi de 23,4 anos (variando de 1,3 a 52 anos de idade) com predomínio do sexo feminino (54\%).

Resultados: As operações realizadas foram: troca de valva aórtica em 9 (25,7\%) pacientes (8 próteses biológicas e uma metálica); troca de valva mitral em $6(17,1 \%)$ casos (em todos utilizou-se próteses biológicas) e uma plastia mitral $(2,9 \%) ; 2(5,8 \%)$ pacientes com troca mitro-aórtica; atrioseptoplastia em $13(37,1 \%)$; ventriculoseptoplastia em 1 (2,9\%); e $3(8,5 \%)$ revascularizações do miocárdio sendo uma sem o auxílio de circulação extracorpórea. A abordagem cirúrgica foi feita por miniesternotomia em "H" em 7 (20\%) casos em "L" em $3(8,5 \%)$ e nos 25 casos restantes via mini-incisão. O tamanho das incisões variou de 7 a $14 \mathrm{~cm}$, com média de $9,9 \mathrm{~cm}$.

Conclusão: Estas duas vias de acesso ao coração na operação cardíaca são perfeitamente viáveis e reprodutíveis, sem alteração no tempo cirúrgico, bem como no tempo de CEC, não acarretando portanto, maiores riscos ao paciente, apresentando efeito estético final melhor do que as esternotomias convencionais.

DESCRITORES: Esterno, cirurgia. Toracotomia, métodos.

\section{INTRODUÇÃO}

A cirurgia cardíaca é uma das especialidades que mais evoluiu nos últimos anos. Acompanhando esta evolução surgiram várias maneiras de abordagem do coração. Inicialmente, MILTON ${ }^{(1)}$, em 1897, descreveu o acesso ao mediastino posterior através da secção vertical do esterno. Posteriormente,
HOLMANN \& WILLET (2), em 1949, procederam a esternotomia vertical parcial com secção ao nível do $2^{\circ}$ espaço intercostal para pericardiectomias. Em 1953, Shumaker e Blalock realizaram esternotomias verticais completas, para respectivamente, a operação de valvotomia pulmonar e os primeiros casos de anastomose subclavio-pulmonar ${ }^{(3)}$. Com a evolução

Trabalho realizado no Instituto Dante Pazzanese de Cardiologia. São Paulo, SP, Brasil.

Recebido para publicação em agosto de 1999.

* Do Instituto Dante Pazzanese de Cardiologia.

Endereço para correspondência: Fabian Castilho. Rua Prudente de Moraes, 1084 - Centro. Ribeirão Preto, SP. Brasil. CEP: 14015-100. Tel: (011) 3086-4517.e-mail: arnoni@br2001.com.br 
Castilho F, Arnoni A S, Arnoni R T, Rivera J A, Almeida A F S, Abdulmassih Neto C, Dinkhuysen J J, Issa M, Chaccur P, Paulista P $\mathrm{P}$ - Miniesternotomia e mini-incisão: experiência inicial do Instituto Dante Pazzanese de Cardiologia. Rev Bras Cir Cardiovasc 2000; 15 (1): 39-43.

da operação e o advento da circulação extracorpórea, a esternotomia passou a ser utilizada de rotina nos procedimentos intracardíacos, conforme relatam JULIAN et al. (3). Na década de 90 , houve uma preocupação de tornar a cirurgia cardíaca menos onerosa e com menor trauma cirúrgico ao paciente. Com esta finalidade passou-se a utilizar a miniesternotomia e a mini-incisão visando diminuir a morbidade do procedimento cirúrgico com melhor efeito estético ${ }^{(4)}$.

O objetivo deste trabalho é avaliar a viabilidade, reprodutibilidade e efeito estético final destas duas abordagens cirúrgicas do coração: a miniesternotomia e a mini-incisão.

\section{CASUÍSTICA E MÉTODOS}

No período de agosto de 1997 a outubro de 1998, 35 pacientes de nosso Serviço foram submetidos a tratamento cirúrgico de seus defeitos cardíacos através da abordagem por miniesternotomia ou mini-incisão.

Houve neste grupo predomínio do sexo feminino (19 pacientes 56\%).

A idade dos pacientes variou de 1 ano e três meses a 52 anos (média de 23,4 anos), com peso médio de $45,7 \mathrm{~kg}$ (7 a $80 \mathrm{~kg}$ ). Várias cardiopatias foram tratadas por estas duas técnicas, conforme apresentado nas Tabelas 1 e 2 .

\section{Técnica Operatória}

A técnica operatória empregada nos dois grupos foi semelhante, variando apenas quanto à via de acesso.

Nos pacientes cujo acesso foi mediante miniincisão, a incisão na pele foi reduzida, seguida de dissecção do tecido celular subcutâneo, com completa exposição do esterno e secção vertical total deste. Já para os casos de miniesternotomia, a incisão reduzida na pele era seguida de secção parcial do esterno, seja em "H" ou em "L" (4-8).

\section{TABELA 1}

CARDIOPATIAS TRATADAS NESTA SÉRIE POR MINIESTERNOTOMIA

\section{DIAGNÓSTICO}

$N^{\circ} D E$ CASOS

\begin{tabular}{lr} 
Defeito do septo interatrial & 2 \\
Afecção valva mitral & 3 \\
Afecção valva aórtica & 5 \\
TOTAL & 10 \\
\hline
\end{tabular}

Todos os pacientes foram operados sob circulação extracorpórea (CEC), com hipotermia moderada, executando-se uma revascularização do miocárdio. O tratamento da afecção da valva mitral foi realizado mediante pinçamento anóxico e demais cardiopatias com cardioplegia cristalóide.

Dois pacientes coronariopatas foram submetidos a revascularização do miocárdio apenas com a anastomose da artéria torácica interna para o ramo interventricular anterior com CEC, cuja proteção miocárdica se deu por intermédio de cardioplegia cristalóide e pinçamento anóxico. O outro paciente foi revascularizado com a artéria torácica interna esquerda para o ramo interventricular anterior e a veia safena para artéria coronária direita, sem CEC.

Em todos os pacientes, optou-se pela canulação da aorta ascendente de maneira semelhante à realizada nas operações de rotina em nossa Instituição. Ambas as cavas foram canuladas, separadamente, para procedimentos sobre a valva mitral, septo interatrial e septo interventricular.

O tratamento das afecções da valva aórtica foi realizado com canulação única do átrio direito, semelhante ao que realizamos nos pacientes que foram revascularizados. Para drenagem das cavidades esquerdas utilizou-se aspirador na emergência da veia pulmonar superior direita.

\section{RESULTADOS}

Não houve intercorrências durante o ato operatório. As operações realizadas estão apresentadas nas Tabelas 3 e 4 .

O tamanho das incisões variou entre 7 e $14 \mathrm{~cm}$, com média de $9,9 \mathrm{~cm}$, que equivale, nesta amostra, a $40 \%$ do tamanho da incisão normal (entende-se por incisão normal, aquela que é feita de rotina em nosso Serviço, estendendo-se da fúrcula até $2 \mathrm{~cm}$ abaixo do apêndice xifóide). As Tabelas 5,6 e 7

TABELA 2

\begin{tabular}{lc}
\multicolumn{2}{c}{ CARDIOPATIAS TRATADAS NESTA SÉRIE } \\
POR MINI-INCISÃO \\
\hline DIAGNÓSTICO & N DE CASOS $^{c}$ \\
\hline Defeito do septo interatrial & 11 \\
Defeito do septo interventricular & 1 \\
Afecção valva mitral & 4 \\
Afecção valva aórtica & 4 \\
Afecção mitro-aórtica & 2 \\
Insuficiência coronariana & 3 \\
\hline TOTAL & 25 \\
\hline
\end{tabular}


Castilho F, Arnoni A S, Arnoni R T, Rivera J A, Almeida A F S, Abdulmassih Neto C, Dinkhuysen J J, Issa M, Chaccur P, Paulista P $\mathrm{P}$ - Miniesternotomia e mini-incisão: experiência inicial do Instituto Dante Pazzanese de Cardiologia. Rev Bras Cir Cardiovasc 2000; 15 (1): 39-43.

TABELA 3

OPERAÇÕES REALIZADAS POR MINIESTERNOTOMIA

\begin{tabular}{lc}
\hline OPERAÇÃO & $N^{\circ}$ DE CASOS \\
\hline Atrioseptoplastia & 2 \\
Troca de valva mitral - prótese biológica & 3 \\
Troca de valva aórtica - prótese biológica & 5 \\
\hline
\end{tabular}

TABELA 4

\begin{tabular}{|c|c|}
\hline \multicolumn{2}{|c|}{ OPERAÇÕES REALIZADAS POR MINI-INCISÃO } \\
\hline OPERAÇÃO & $N^{\circ}$ DE CASOS \\
\hline Atrioseptoplastia * & 11 \\
\hline Ventriculoseptoplastia & 1 \\
\hline Troca de valva mitral - prótese biológica & 3 \\
\hline Troca de valva aórtica - prótese biológica & 3 \\
\hline Troca de valva aórtica - prótese metálica ** & 1 \\
\hline Dupla troca mitro-aórtica - prótese biológica & 2 \\
\hline Plastia mitral & 1 \\
\hline Revascularização do miocárdio & 3 \\
\hline \multicolumn{2}{|c|}{$\begin{array}{l}\text { * } 1 \text { caso: defeito associado: Drenagem anômala da veia } \\
\text { pulmonar superior direita. } \\
{ }^{*} \text { Associado à ampliação do anel aórtico pela Técnica de } \\
\text { Manoguian }{ }^{(9)} \text {. }\end{array}$} \\
\hline
\end{tabular}

mostram uma comparação entre o tempo de CEC e anoxia, de pacientes operados pela técnica convencional com aquele abordados por miniesternotomia e mini-incisão. Foram selecionados, para este estudo, pacientes submetidos a tratamento cirúrgico no mesmo período e portadores de cardiopatias semelhantes.

Não houve diferença significativa, tanto no tempo de CEC, quanto no de anoxia. Apenas nos pacientes submetidos a procedimentos sob a valva mitral aparentemente há um menor tempo de anoxia, naqueles submetidos ao acesso por mini-incisão e miniesternotomia.

Na evolução pós-operatória, um paciente apresentou sangramento aumentado no pós-operatório imediato, sendo submetido a revisão cirúrgica da hemostasia, havendo necessidade de aumentar a incisão da pele de 9 a $13 \mathrm{~cm}$. O paciente evoluiu bem no pós-operatório, recebendo alta como os demais. Não houve óbito nesta casuística.

O tempo de internação foi semelhante ao dos pacientes operados de forma convencional em nosso Serviço.

\section{COMENTÁRIOS}

Com o avanço dos métodos hemodinâmicos para correção de defeitos cardíacos, depara-se o cirurgião com a exigência atual em realizar operações que possibilitem a redução da morbi-mortalidade e apresentem melhor resultado estético (8).

A operação cardíaca com abordagem do coração por mini-incisão e miniesternotomia mostra-se como uma boa alternativa (4-11), sem a necessidade de material específico. Além disso, não há aumento do tempo cirúrgico total, bem como do tempo de CEC e anoxia, o que torna este método viável.

Na mini-incisão o controle do campo operatório é total, e uma vez havendo a necessidade de um maior campo decorrente de qualquer complicação na operação, este é factível com a ampliação da incisão na pele o que torna este método de abordagem do coração mais seguro quando comparado a outros.

Esse tipo de acesso é facilmente reprodutível e permite a perfeita visualização das estruturas cardíacas, possibilitando a correção de defeitos dos átrios e da aorta.

TABELA 5

\begin{tabular}{|c|c|c|c|c|}
\hline \multicolumn{5}{|c|}{ COMPARAÇÃO ENTRE TEMPO DE CEC E ANOXIA (MINUTOS) } \\
\hline AÓRTICO & MÉDIA & MEDIANA & MÍNIMO & MÁXIMO \\
\hline \multicolumn{5}{|c|}{ Convencional } \\
\hline CEC & 65,63 & 67,50 & 40,00 & 85,00 \\
\hline Anoxia & 49,13 & 50,00 & 28,00 & 67,00 \\
\hline \multicolumn{5}{|c|}{ Miniesternotomia+ mini-incisão } \\
\hline CEC & 60,13 & 60,00 & 50,00 & 75,00 \\
\hline Anoxia & 41,63 & 41,50 & 33,00 & 48,00 \\
\hline $\begin{array}{l}\text { Análise esta } \\
\text { CEC } \\
\text { ANOXIA }\end{array}$ & $\begin{array}{l}\text { atística: Teste Mann-Whitney } \\
p=0,2786 \\
p=0,1049\end{array}$ & & & \\
\hline
\end{tabular}


Castilho F, Arnoni A S, Arnoni R T, Rivera J A, Almeida A F S, Abdulmassih Neto C, Dinkhuysen J J, Issa M, Chaccur P, Paulista P P - Miniesternotomia e mini-incisão: experiência inicial do Instituto Dante Pazzanese de Cardiologia. Rev Bras Cir Cardiovasc 2000; 15 (1): 39-43.

TABELA 6

\begin{tabular}{lcccc}
\hline \multicolumn{4}{c}{ COMPARAÇÃO ENTRE TEMPO DE CEC E ANOXIA (MINUTOS) } \\
\hline MITRAL & MÉDIA & MEDIANA & MíNIMO & MÁXIMO \\
\hline Convencional & & & 55,00 & 75,00 \\
CEC & 64,33 & 65,50 & 41,00 & 64,00 \\
Anoxia & 48,33 & 43,50 & 40,00 & 70,00 \\
Miniesternotomia+mini-incisão & 50,00 & 45,00 & 28,00 & 43,00 \\
CEC & 34,75 & 34,00 & & \\
Anoxia & & & & \\
\hline
\end{tabular}

Análise estatística: Teste Mann-Whitney

CEC $\quad p=0,1143$

Anoxia $\quad p=0,0667$

TABELA 7

COMPARAÇÃO ENTRE TEMPO DE CEC E ANOXIA (MINUTOS)

\begin{tabular}{lcccc}
\hline CIA & MÉDIA & MEDIANA & MÍNIMO & MÁXIMO \\
\hline Convencional & & & & 75,00 \\
CEC & 44,44 & 45,00 & 20,00 & 55,00 \\
Anoxia & 32,00 & 33,00 & 12,00 & \\
Miniesternotomia+mini-incisão & & & & \\
CEC & 41,43 & 40,00 & 20,00 & 60,00 \\
Anoxia & 28,43 & 30,00 & 14,00 & 42,00 \\
\hline
\end{tabular}

Análise estatística: Teste Mann-Whitney

CEC $\quad p=0,9182$

Anoxia $\quad p=0,6065$

Através do átrio direito podemos corrigir comunicação interatrial e pela valva tricúspide as comunicações interventriculares. A boa visibilização dos defeitos cardíacos pode ser comprovada pelo tempo de circulação extracorpórea, que é semelhante ao do grupo de pacientes com incisão convencional.

Portanto, estas duas formas de abordagem do coração mostram-se passíveis de realização, tra- zendo, em nossa opinião, como principal contribuição um efeito estético melhor, sem colocar em risco o paciente e sem a necessidade de aparelhos ou instrumental que aumentem o custo do procedimento. É nossa prioridade, após estes casos o desenvolvimento e o aprimoramento da técnica de miniincisão a qual consideramos melhor, pois sua reversão é mais fácil. 
Castilho F, Arnoni A S, Arnoni R T, Rivera J A, Almeida A F S, Abdulmassih Neto C, Dinkhuysen J J, Issa M, Chaccur P, Paulista P $\mathrm{P}$ - Miniesternotomia e mini-incisão: experiência inicial do Instituto Dante Pazzanese de Cardiologia. Rev Bras Cir Cardiovasc 2000; 15 (1): 39-43.

RBCCV 44205-485

Castilho F, Arnoni A S, Arnoni R T, Rivera J A, Almeida A F S, Abdulmassih Neto C, Dinkhuysen J J, Issa M, Chaccur P, Paulista P P - Initial study in ministernotomy and mini-incision Dante Pazzanese Institute of Cardiology. Rev Bras Cir Cardiovasc 2000; 15 (1): 39-43.

ABSTRACT: Objective: Ministernotomy and mini-incision are options to approach the heart. The latter, is a small incision on the skin with complete division of the sternum. The goal of this study was to evaluate the two approaches regarding viability, reproducibility and the final estetic effect.

Material and Methods: "H" and "L" miniesternotomies and mini-incision surgeries had been realized for the treatment of valve defects (mitral and aortic), congenital heart defects (IAC and IVC) and three myocardial revascularizations. Thirty-five pacients were assessed: 10 submitted to ministernotomy $(40 \%)$ and 24 to miniincision (60\%). Average age was 23.4 years (range 3 months to 52 years) with females predominating (54\%).

Results: The surgeries included: implant of aortic prostetic valve $-9(25.7 \%), 8$ were biological valves and one a mechanical valve; implant of mitral valve $-6(17.1 \%)$ utilizing biological valves and one plastic mitral valve (2.9\%); two mitro-aortic valve replacements (5.8\%), correction of atrial septal defect $13(37.1 \%)$ and correction of ventricular septal defect, $1(2.9 \%)$ and $3(8.5 \%)$ myocardial revascularizations, one without extracorporeal circulation. " $\mathrm{H}$ " ministernotomy had been done in $7(20 \%)$ cases and in "L" in $3(8.5 \%)$ cases and via mini-incision in the remaining cases (25). The size of the incisions ranged from 7 to $14 \mathrm{~cm}$ with an avarege of $9.9 \mathrm{~cm}$.

Conclusion: This two approaches to the heart for cardiac surgery are perfectly viable and reproducible without changing surgical time, or extracorporeal circulation time without greater risks to the patient, and resulting in a better final estetic effect than conventional sternotomy.

DESCRIPTORS: Sternotomy, surgery. Thoracotomy, methods.

\section{REFERÊNCIAS BIBLIOGRÁFICAS}

1 Milton $\mathrm{H}$ - Mediastinal surgery. Lancet 1897; 1: 872-5.

2 Holmann E \& Willet F - The surgical correction of constrictive pericarditis. Surg Gineco \& Obst, 1949; 89: 129-44.

3 Julian O C, Lopez-Belio M, Dye W S et al. - The median sternal incision in intracardiac surgery with extracorporeal circulation: a general evaluation of its use in heart surgery. Surgery 1957; 42: 753-61.

4 Mulinari L A, Tyszka A L, Costa F D A et al. Miniesternotomia: um acesso seguro para a cirurgia cardíaca. Rev Bras Cir Cardiovasc 1997; 12: 335-9.

5 Cosgrove D M \& Sabik J F - Minimally invasive approach for aortic valve operations. Ann Thorac Surg 1996; 62: 596-7.

6 Loulmet D F, Carpentier A, Cho P W et al. - Less invasive technique for mitral valve surgery. J Thorac Cardiovasc Surg 1998; 115: 772-9.
$7 \quad$ Konertz W, Waldenberger F, Schmutzler M, Ritter J, Liu $\mathrm{J}$ - Minimal access valve surgery through superior partial sternotomy: a preliminary study. $J$ Heart Valve Dis 1996; 5: 638-40.

8 Wilson Jr. W R, Ilbawi M N, DeLeon S Y, Piccione Jr. W, Tubeszewski K, Cutilletta A F - Partial median sternotomy for repair of heart defects: a cosmetic approach. Ann Thorac Surg 1992; 54: 892-3.

9 Manouguian S \& Seybold-Epting W - Patch enlargement of the aortic valve ring by extending the aortic incision into the anterior mitral leaflet: new operative technique. J Thorac Cardiovasc Surg 1979; 78: 402-12.

10 Doty D B, DiRusso G B, Doty J R - Full-spectrum cardiac surgery through a minimal incision: mini-sternotomy (lower half) technique. Ann Thorac Surg 1998; 65: 573-7.

11 Hirose H, Umeda S, Mori Y, Murakawa S, Azuma K, Hashimoto T - Another approach for aortic valve replacement through left thoracotomy. Ann Thorac Surg 1994; 58: 884-6. 